\title{
III. MEMÓRIA
}

\section{Aidyl Macedo de Queiroz Pérez-Ramos, em hospitais, desertos e academias}

\author{
Aidyl Macedo de Queiroz Pérez-Ramos, in hospitals, deserts and academies \\ Aidyl Macedo de Queiroz Pérez-Ramos, en hospitales, desiertos y academias \\ Doris Lieth Peçanha (CAd. I 9) ${ }^{5}$
}

Resumo: No centésimo número deste periódico, este ensaio faz homenagem a sua idealizadora e Editora, a professora Aidyl, resgatando a memória dessa pioneira em psicologia no Brasil e que foi uma das fundadoras da Academia Paulista de Psicologia. O Boletim da Academia Paulista de Psicologia (APP) figura como exemplo de resiliência, na área editorial, situando-se entre as publicações mais antigas e sistemáticas do continente sul americano. Aidyl também lançou as bases da psicossomática e da psicologia hospitalar no Brasil. É uma das referências nacionais em educação especial, com larga atuação como psicóloga, professora e pesquisadora no Brasil e no exterior, em lugares tanto distantes como as Ilhas Canárias e o deserto do Saara onde atuou como perito das Nações Unidas.

Palavras-chave: história da psicologia; psicologia clínica; psicologia hospitalar; educação especial; editor.

\begin{abstract}
In the 100th issue of this journal, this essay pays homage to its creator and editor, professor Aidyl, rescuing the memory of this pioneer in Psychology in Brazil and one of the founders of the São Paulo Academy of Psychology. The Bulletin of the São Paulo Academy of Psychology (APP) is an example of resilience in the editorial area, being among the oldest and most systematic publications in the South American continent. Aidyl also launched the bases of psychosomatics and hospital psychology in Brazil. She is one of the national references in special education, with extensive experience as a psychologist, teacher, and researcher in Brazil and abroad, in places as far as the Canary Islands and the Sahara desert, where she worked as a United Nations expert.
\end{abstract}

Keywords: history of psychology; clinical psychology; hospital psychology; special education; editor.

Resumen: En el número 100 de esta revista, este ensayo rinde homenaje a su creador y editor, el profesor Aidyl, rescatando la memoria de este pionero de la psicología en Brasil y que fue una de los fundadores de la Academia Paulista de Psicología. El Boletín de la Academia Paulista de Psicología (APP) es un ejemplo de resiliencia en el ámbito editorial, siendo una de las publicaciones más antiguas y sistemáticas del continente sudamericano. Aidyl también lanzó las bases de la psicología psicosomática y hospitalaria en Brasil. Es una de las referencias nacionales en educación especial, con una amplia experiencia como psicóloga, profesora e investigadora en Brasil y en el extranjero, en lugares tan distantes como las Islas Canarias y el desierto del Sahara, donde sirvió como experta de las Naciones Unidas.

Palabras clave: historia de la psicología; psicología clínica; psicología hospitalaria; educación especial; editor.

\section{Introdução}

O centésimo número deste periódico remete-nos a sua idealizadora e Editora por aproximadamente quatro décadas, a Profa. Titular em Psicologia, Dra. Aidyl Macedo de Queiroz Pérez-Ramos. Para tanto, Aidyl inspirou-se na editoração científica norte-americana, notadamente no Academy Update, da The New York Academy of Sciences. Na América Latina é grande a mortalidade das revistas de psicologia, sendo poucas aquelas que sobrevivem por mais de trinta anos. Pois o Boletim da Academia Paulista de Psicologia (APP) figura como exemplo de resiliência, na área editorial, situando-se entre as publicações mais longevas e sistemáticas do continente sul americano. Isso se deve, em grande medida, ao incansável trabalho de Aidyl que manteve a periodicidade dessa revista científica, publicação oficial da APP e, ainda hoje, compõe seu corpo editorial. Portanto, este texto, visa resgatar um pouco da história dessa psicóloga como forma de lhe homenagear e agradecer. Os nomes Aidyl, Boletim e Academia figuram intrinsecamente

${ }^{51}$ Psicóloga, Doutorado (IPUSP/Sorbonne) e Pos-doutorados (Canadá e França) em psicologia clínica, Profa Titular Sênior na Unidade Saúde Escola da Universidade Federal de São Carlos (UFSCar), São Carlos, São Paulo. Coordenadora do Grupo de Pesquisa VIDA - Vivência Intrapsíquica e Desenvolvimento Ambiento-Organizacional (CNPq-Br). Cad. nº 19 "Pedro de Alcântara Machado" da Academia Paulista de Psicologia, São Paulo (APP-SP). Contato: E-mail: pecanhadl@yahoo. com ORCID 0000-0002-8262-3775. 
ligados em nossa memória. Num pensamento circular, escrever sobre Aidyl é também fazer referência à APP que ela ajudou a fundar (1979), sendo membro de sua diretoria, na qualidade de secretária geral, por décadas. Aidyl é titular da Cadeira Geraldo Horácio Paula de Souza (no 30), ocupando, atualmente, o cargo honorífico de "Presidente do Passado".

A Dra. Aidyl, ou simplesmente Aidyl para seus muitos amigos, é acadêmica em psicologia clínica, educação especial e história da psicologia, com longa atuação neste país e em vários outros do continente americano, da África e Europa. Pioneira em psicologia hospitalar pediátrica, foi também "Perito" em psico-educação em programas de desenvolvimento humano nacionais e internacionais. Foi a primeira psicóloga brasileira a trabalhar na ONU (1970; Leal, 2006), atuando nos projetos: "SPA-70" (ONU: Ilhas Canárias, Espanha e Saara Ocidental, África); Multinacional de Educação (OEA: Brasil, Paraguai e Uruguai) e Projeto Família (UNICEF: Venezuela).

No âmbito da docência em psicologia, Aidyl contribuiu em universidades do estado de São Paulo (Pontifícia Universidade Católica de São Paulo-PUC-SP; Univ. Estadual Paulista UNESP- campus Assis; Univ. Estadual de Campinas - Unicamp e Univ. de São Paulo, USP-SP) e no exterior (Profa. Visitante: Univ. Central de Venezuela, Venezuela, em 1963, 1973, 1979-1986; Univ. La Laguna, Espanha, 1978 e em outras oportunidades); em pesquisa-intervenção com apoio de organismos de fomento científico no Brasil (FAPESP, CAPES e CNPq) e em publicações que ultrapassam uma centena, com reconhecimento nacional e internacional (Kay, 1988).

Quanto ao aspecto pessoal, nossa homenageada nasceu na pequena cidade de Ribeirão Bonito, no estado de São Paulo (Br.), aos 14 de março de 1925. Filha primogênita de Romilda Macedo de Queiroz e Sebastião Queiroz, bacharel em direito e tabelião, teve apenas um irmão igualmente bacharel em direito e já falecido. Casou-se (1964) com o psicólogo, economista e também membro titular da APP, o Prof. Dr. Juan Pérez-Ramos (The American Biographical Institute, 1991, p. 240) nascido em La Palma (1918, Espanha). Com ele viveu harmoniosamente e desenvolveu parceria científica (Pérez-Ramos, A., \& Pérez-Ramos, J., 1978 e 1996) até o falecimento de Juan na cidade de São Paulo (Peçanha, 2006a).

Aidyl possui graduação em Educação pela Faculdade de Filosofia (USP-SP, 1943-1948). Foi aluna de eminentes professores vindos da Europa (aulas em francês) e dos EUA para colaborar com a formação intelectual naquele meio emergente. Realizou especializações em psicologia na Universidade do Chile (UC, 1948 e 1950), na Sorbonne (Paris, 1951) e nas universidades de Harvard, Ohio e Columbia (EUA). Participou do $1^{\circ}$ Congresso em Psiquiatria e Criminologia em Paris (1950), presentes Ana Freud e Melanie Klein (Pérez-Ramos, 2006). Durante sua estadia na Europa, por meio de bolsa de estudos, Aidyl também participou de atividades no Instituto Jean Jacques Rousseau (Suíça). Graduou-se em Psicologia pela Univ. de Columbia (USA,1956). Doutorou-se em Psicologia Clínica (PUC-SP, 1956-1960) com a tese "Os desajustamentos da criança asmática: uma contribuição à psicologia clínica quanto ao seu conteúdo e às suas técnicas" (Queiroz, 1960) que inspirou (Peçanha, Pérez-Ramos, \& Lacharité, 2003; Peçanha, \& Lacharité, 2007) e ainda surpreende pela profundidade e rigor clínico teórico-metodológico. Nela estudou aspectos emocionais da criança com asma, utilizando testes intelectuais e técnicas projetivas, como o Rorschach e o CAT, num delineamento quase experimental e misto de pesquisa (111 casos clínicos e tratamento estatístico em 3 grupos com 37 crianças cada um; orientador: Enzo Azzi, italiano, ex-diretor do Instituto de Psicologia - IPPUC-SP). Aidyl realizou seu Pós-doutorado na Universidade de Columbia (EUA, 1968) e residência médica no Centro de Atendimento ao Estudante da Northern Illinois University (EUA, 1978).

Assessorou governos no Brasil e na Espanha (anos 70), oferecendo o conhecimento necessário para a criação de associações em educação especial no Brasil (notadamente em São Paulo, SP, e em Curitiba, Pr.), na Venezuela (Associação Venezuelana de Pais e Amigos dos Excepcionais, em Caracas) e na Espanha. Implementou programa pioneiro em atenção integral ao deficiente mental nas Ilhas Canárias, reconhecido pelos jornais da época (La Província,1971) como o primeiro de toda a Espanha, legando centros modelos em psico-educação para esse país, e quiçá para a Europa, como referido naquela reportagem.

Aidyl foi pioneira no desenvolvimento e utilização de um sistema transacional de avaliação-intervenção psicológica (Pérez-Ramos, 1990) numa época em que ainda predominavam os modelos lineares. Ou seja, por meio dele são integradas as fases de diagnóstico e tratamento clínico ou psico-educativo. Orientou dezenas de teses, em especial no programa de pós-graduação em Psicologia Clínica do Instituto 
de Psicologia da Univ. de São Paulo (IP-USP a partir de 1979). Provavelmente orientou o primeiro doutorado na modalidade estudo "sanduíche" (Brasil-França, 1993-1997) em psicologia clínica naquele Instituto (Peçanha, 2015), em parceria com outra pioneira, a Profa. Rosine Debray (Sorbonne, Paris V), uma das fundadoras da Escola Psicossomática de Paris.

Ao longo de sua carreira, Aidyl primou pelo atendimento psicológico empático, nas suas palavras, "humanizado" e "contextualizado", considerando as necessidades das pessoas e grupos nos diversos locais e países em que trabalhou. Notabilizou-se por favorecer condições de desenvolvimento psicológico para populações em situação de grande vulnerabilidade como em regiões do Saara (África), considerado o deserto mais quente do mundo. Assim, conquistou homenagens pelos serviços prestados tanto aos centros de atendimento a crianças nos campos petroleiros da Venezuela, quanto às tribos berberes da região norte da África, quando em missão pela ONU (1974). Destes últimos recebeu vaso de prata incrustado com arabescos próprios àquela cultura. Trata-se de um dos povos mais antigos daquele continente, autodenominados imazighen, ou homens livres. Outra honraria foi o jameo del agua como Aidyl carinhosamente o denomina, representação em ouro de um caranguejo cego, pelo seu trabalho em prol das pessoas com deficiência. Trata-se de uma espécie endêmica de grande interesse científico que habita as profundezas do lago vulcânico los Jameos del Agua (Lanzarote, Espanha, 1973). Tivemos a oportunidade de percorrer os caminhos de Aidyl pelas diferentes ilhas que compõem o arquipélago das Canárias e por essa região do Magreb, ao noroeste do continente africano, cuja palavra árabe significa onde o sol se põe. Certamente Aidyl teve aí uma experiência única e uma vivência que marcou sua vida, aprimorando sua sensibilidade aos contextos e seu respeito pela individualidade de cada pessoa.

Aidyl contribuiu nos primórdios da psicossomática nacional (Queiroz, \& Strauss,1958a) e internacional (Queiroz, \& Strauss, 1958b) por meio de um trabalho pioneiro realizado em conjunto com a medicina. Nas inúmeras conversas que tivemos, ela sempre destacou, com gratidão, o papel de seus pares nessa trajetória, como o entusiasmo e abertura do Dr. Pedro de Alcântara Machado, então Diretor da Clínica Pediátrica da USP, patrono da Cadeira no 19 (APP) que tenho a honra de ocupar, precedida pelo saudoso César Ades. Aidyl lançou as bases para a psicologia hospitalar pediátrica (1956-1961), de caráter interdisciplinar, envolvendo também o contexto familiar dessas crianças na referida clínica da Faculdade de Medicina da USP (atual Instituto da Criança, Hospital das Clínicas HC-SP, SP). A visão "sociobiopsicológica" (Pérez-Ramos, 2006, p.38) e a avaliação-intervenção integradas que, já naquela época, fundamentavam o trabalho de Aidyl, foram decisivas para a expansão desse atendimento a pacientes de outras clínicas daquele Hospital. Houve casos raros, atendidos por Aidyl, aos quais ela costuma fazer referência como o das gêmeas siamesas em que a difícil opção pela vida de uma das meninas se impôs.

Juntamente com os Profs. Enzo Azzi, Aniela Ginsberg e Ana Poppovic, Aidyl participou da criação dos primeiros cursos de especialização em psicologia, oferecidos pela então Faculdade São Bento (da Univ. PUC-SP),nas áreas de Psicologia Clínica, Psicologia da Educação e Psicologia do Trabalho com dois anos de duração e com um semestre de estágio supervisionado. Nessa mesma universidade, Aidyl foi uma das fundadoras da Clínica Psicológica (1959), recebendo o "Troféu aos Fundadores" aos 40 anos dessa Clínica, posteriormente (1993) denominada Ana Maria Poppovic, em homenagem póstuma a sua colega e primeira diretora.

Membro das Associações: Psicologia de São Paulo (antiga Sociedade de Psicologia de São Paulo ( $1^{\mathrm{a}}$. do Brasil, fundada em 09.11.1945), American Psychological Association, Interamerican Society of Psychology, vitalícia na The New York Academy of Sciences (1994) e na APP, o nome de Aidyl figura no The world who's who of women (Kay, 1988). Sua experiência com academias e acadêmicos (em outras áreas no caso brasileiro, dado o pioneirismo da APP), seu amor à ciência, à história, seu gosto e expertise no ato de escrever foram decisivos para que a Profa. idealizasse a revista da APP, o presente Boletim. Há periódicos acadêmicos que privilegiam informar quanto às realizações do próprio sodalício, de seus membros titulares e veicular publicações dos mesmos; outros visam publicar artigos relevantes na área do conhecimento em que se situa a entidade, independentemente da afiliação de seus autores. Por cerca de duas décadas, Aidyl liderando a editoração do Boletim, buscou manter esse formato híbrido, mas acabou cedendo às demandas no sentido de excluir a divulgação das atividades dos acadêmicos. Isso também respondia a uma adaptação aos novos tempos virtuais em que os curricula 
vitae tornaram-se disponíveis on line, a exemplo da plataforma Lattes (sítio CNPq). Assim, o Boletim concentrou-se mais na publicação científica em psicologia e, indexado em várias bases, gradativamente, foi deixando a versão impressa, encontrando-se disponível on-line desde o ano de 2003.

Dedicando-se à preservação da memória da psicologia no Brasil (Pérez-Ramos, 2012), Aidyl e colegas, em parceria com a coordenação da Biblioteca Virtual em Saúde - Psicologia Brasil (BVS-Psi Brasil, referência na América Latina), participou do desenvolvimento do módulo História da Psicologia (Academia Paulista de Psicologia, 2010). São notáveis suas contribuições em estudos de casos (Queiroz, \& Popovic, 1957; Pérez-Ramos, 2002), técnicas projetivas (Queiroz, 1955; Pérez-Ramos, 1966) e outras medidas. Contribuiu para a tradução e adaptação do Coping Responses Inventory for Work Settings de Moos e Schaefer (1993), com o título de Inventário sobre Superação do Stress Profissional (ISSP - Pérez-Ramos, J. \& Pérez-Ramos, A.,1998), retrabalhado e publicado, por meio de pesquisa empírica, como Inventário de Respostas de Coping no Trabalho (IR-
C-T, Peçanha, 2006). Aidyl produziu vasto material para a estimulação precoce e escalas para avaliação do desenvolvimento infantil (Pérez-Ramos, A., \& Pérez-Ramos, J., 1996; Pérez-Ramos, A., 2000), incluindo crianças cegas (Silva, Costa, \& Pérez-Ramos, A., 2018). Seu trabalho é considerado uma referência nacional em educação especial e direitos da criança.

A história da Psicologia brasileira encontra em Aidyl uma das mais produtivas e originais acadêmicas. Com quase um século de vida, orienta trabalhos, dedica-se à APP, participa de bancas e de reuniões virtuais (motivo Covid-19). Fora desta situação pandêmica, manteve-se, com sua gentileza habitual, disponível para um cafezinho com as visitas, apreciando saber das "novidades" e partilhar suas muitas memórias e conhecimentos. Amigos e orientados consideram-na uma enciclopédia vida e expressam sua gratidão à "Mestra" generosa e acolhedora que alavancou muitas vidas, com fé no exercício profissional, alicerçado na ética e em sólida formação. "Tenham sempre como prioridade o bem-estar do ser humano nos mais variados contextos" (Pérez-Ramos, 2006, p. 42).

\section{Referências}

Academia Paulista de Psicologia (2010). O Legado da Psicologia para o Desenvolvimento Humano. 2a. fase. Bauru: Gráfica Coelho.

Kay, E. (1988). The world who's who of women. (Aidyl Macedo de Queiroz Pérez-Ramos pp.540-541). 9a ed. Cambridge, England: International Biographical Center (1973).

La Província (1971). El Centro de Educación Especial de Tafira, modelo para a Europa. Miércoles, 09 de junio.

Leal, G. (2006). Persona: Aidyl Pérez-Ramos. Entre hospitais e desertos. Viver Mente \& Cérebro, 14(159), 22-24.

Moos, R.H., \& Schaefer, J.A. (1993). Coping responses inventory for work settings (CRI-Form W). HSR \& D Center for Health Care Evaluation, Stanford University and Department of Veterans Affairs Medical Center. Palo Alto, CA. USA (Manuscrito cedido ao casal Pérez-Ramos).

Peçanha, D. L. (2006a). Obituário Juan Pérez-Ramos. Boletim Academia Paulista de Psicologia, São Paulo, 2(6), $107-111$.

Peçanha, D. L. (2006b). Avaliação do coping numa equipe de enfermagem oncopediátrica. Boletim Academia Paulista de Psicologia, São Paulo, 2(6), 69-88.

Peçanha, D.L. (2015). A criança com asma e sua família: avaliação psicossomática e sistêmica. São Carlos: EdUFSCAR (apoio FAPESP).

Peçanha, D.L., \& Lacharité, C. (2007). The family systemic assessment: un study about its construct validity with asthmatic and healthy children and their families. Psicologia em Estudo, 2(3), 503-512.

Peçanha, D.L, Pérez-Ramos, A.M.Q., \& Lacharité, C. (2003). Une méthode systémique d'évaluation du fonctionnement familial: étude portant sur les familles des enfants asthmatiques. Revue Québécoise de Psychologie, 24(2), 89-112.

Pérez-Ramos, A.M.Q. (1966). Psicología clínica: técnicas de diagnóstico. Madri, Espanha: Mediterráneo.

Pérez-Ramos, A. M. Q. (1978). Estimulação precoce: informações básicas aos pais e profissionais. Brasília, Ministério da Educação e Cultura e Organização dos Estados Americanos, Departamento de Documentação e Divulgação (Projeto Especial Multidisciplinar de Educação Brasil-Paraguai-Uruguai).

Pérez-Ramos, A.M.Q. (1990). Modelos de prevenção: perspectivas dos programas de estimulação precoce. Psicologia USP 1(1), 6775.

Pérez-Ramos, A.M.Q. (2000). Avaliação prospectiva: o exame precoce da criança. In Jurema Alcides Cunha (Org.) Psicodiagnóstico V (5.ed.). (pp.151-157). Porto Alegre: Artes Médicas Sul.

Pérez-Ramos, A.M.Q. (2006). Humanização hospitalar: conquistas e desafios na realidade brasileira. In A.L. Angelini, \& A.M.Q. Pérez-Ramos et al. (Org.). O Legado da Psicologia para o Desenvolvimento Humano. Resgate da vida e obra de acadêmicos titulares através de depoimentos e DVDs. (pp.36-45). Bauru: Gráfica Coelho. 
Pérez-Ramos, A.M.Q. (2012). Helena Wladermina Antipoff, un héritage importante (25/03/1892-09/08/1974) (P. Duque-Estrada, Trad.) In: Hebe C.B.A. Costa. Elles sont venues de loin... ces femmes, sources d'inspiration pour le Brésil. (pp. 197-202). France: Yvelinédition.

Pérez-Ramos, A.M.Q. (2020). Memórias da psicologia no Brasil. Boletim Academia Paulista de Psicologia, 40(99), 290.

Pérez-Ramos, A. M. Q., \& Maia, E.M.C. (2002). O comportamento de apego em pesquisas longitudinais de estudos de casos. Boletim de Psicologia, 52(116), 29-43.

Pérez-Ramos, A.M.Q., \& Pérez-Ramos, J. (1996). Estimulação precoce: serviços, programas e currículos. $3^{3}$ ed. Brasília: CORDE.

Pérez-Ramos, J., \& Pérez-Ramos, A. (1998). Inventário sobre superação do stress profissional - ISSP, In: D.P.S.A. Ribeiro (1999). Estratégias de "coping" em psicólogos dos serviços básicos e ambulatoriais de saúde pública. (Anexo I. Dissertação de Mestrado não publicada. Instituto de Psicologia Universidade de São Paulo, São Paulo, Brasil).

Pérez-Ramos, A. M. Q., \& Pérez-Ramos, J. (1978). Estimulação precoce. Ministério da Educação e Cultura (Projeto Especial Multidisciplinar de Educação Brasil-Paraguai-Uruguai).

Queiroz, A.M. (1955). Motivos ornamentais em desenhos de crianças. Revista de Psicologia Normal e Patológica, I(2), $370-381$.

Queiroz, A.M. (1960). Contribuições psicológicas à clínica da criança asmática. Revista de Psicologia Normal e Patológica. São Paulo (número especial).

Queiroz, A.M., \& Popovic, A. M. (1957). Considerações sobre a ludoterapia. Evolução de dois casos. Revista de Psicologia Normal e Patológica, II (2), 264-294.

Queiroz, A.M., \& Strauss, A. (1958a). Contribuições psicológicas à clínica da criança asmática. Revista Psicológica Normal e Patológica, IV, 83-94.

Queiroz, A.M., \& Strauss, A. (1958b). Some psychological contributions to the clinical treatment of the asthmatic child. Acta Allergologica, USA, XII, 396-406.

Silva, S. M. M., Costa, M. P. R., \& Pérez-Ramos, A. M. Q. (2018). Brincadeiras para crianças com deficiência visual. Curitiba: Editora Appris. The American Biographical Institute (1991). The International Book of Honor, $3^{\text {a }}$ ed. (Juan Pérez-Ramos, p. 240). Carolina do Norte, USA: Author.

The New York Academy of Sciences - 1817 (1994). Directory of Members. (Aidyl Macedo de Queiroz Pérez-Ramos, p. 272). New York: Harris. 